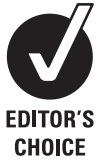

\section{See Commentary, p 1442}

${ }^{1}$ Department of Pediatric and Adolescent Medicine, Division of Gastroenterology and Hepatology, Mayo Clinic, Rochester, Minnesota, USA;

${ }^{2}$ Department of Internal

Medicine, Division of

Gastroenterology and Hepatology, Mayo Clinic

Rochester, Minnesota, USA;

${ }^{3}$ Department of Health Sciences

Research, Division of

Biostatistics, Mayo Clinic,

Rochester, Minnesota, USA

Correspondence to:

Dr P Angulo, Division of Digestive Diseases and

Nutrition, University of Kentucky, 800 Rose Street, Rm MN469,

Lexington, KY 40536, USA;

paul.angelo@uky.edu

AF is now at the Cleveland Clinic Foundation, Pediatric

Gastroenterology and Cell

Biology, Cleveland, Ohio, USA.

Revised 22 February 2009

Accepted 1 April 2009

Published Online First

21 July 2009

\title{
The natural history of non-alcoholic fatty liver disease in children: a follow-up study for up to 20 years
}

\author{
A E Feldstein, ${ }^{1}$ P Charatcharoenwitthaya, ${ }^{2}$ S Treeprasertsuk, ${ }^{2} \mathrm{~J}$ T Benson, ${ }^{3}$ F B Enders, ${ }^{3}$ \\ P Angulo ${ }^{2}$
}

\begin{abstract}
Objectives: The long-term prognosis of non-alcoholic fatty liver disease (NAFLD) in children remains uncertain We aimed at determining the long-term outcomes and survival of children with NAFLD.
\end{abstract}

Design: Retrospective longitudinal hospital-based cohort study.

Patients: Sixty-six children with NAFLD (mean age 13.9 (SD 3.9) years) were followed up for up to 20 years with a total of 409.6 person-years of follow-up.

Results: The metabolic syndrome was present in 19 $(29 \%)$ children at the time of NAFLD diagnosis with 55 (83\%) presenting with at least one feature of the metabolic syndrome including obesity, hypertension, dyslipidaemia and/or hyperglycaemia. Four children with baseline normal fasting glucose developed type 2 diabetes 4-11 years after NAFLD diagnosis. A total of 13 liver biopsies were obtained from five patients over a mean of 41.4 (SD 28.8) months showing progression of fibrosis stage in four children. During follow-up, two children died and two underwent liver transplantation for decompensated cirrhosis. The observed survival free of liver transplantation was significantly shorter in the NAFLD cohort as compared to the expected survival in the general United States population of the same age and sex (log-rank test, $p<0.00001)$, with a standardised mortality ratio of 13.6 (95\% confidence interval, 3.8 to 34.8$)$. NAFLD recurred in the allograft in the two cases transplanted, with one patient progressing to cirrhosis and requiring re-transplantation.

Conclusions: Children with NAFLD may develop endstage liver disease with the consequent need for liver transplantation. NAFLD in children seen in a tertiary care centre may be associated with a significantly shorter survival as compared to the general population.

Non-alcoholic fatty liver disease (NAFLD) is the most common cause of chronic liver disease in the pre-adolescent and adolescent age groups in most of the Western world. An autopsy study found that $9.6 \%$ of the American population aged 219 years have NAFLD, and this figure increased to $38 \%$ among those who were obese. ${ }^{1}$ Similar high figures have been reported among children from countries in Europe and Asia. ${ }^{2-4}$ Insulin resistance is almost a universal finding in paediatric NAFLD and, consequently, several of the clinical features associated with insulin resistance such as obesity, diabetes mellitus and dyslipidaemia are common comorbidities in children who suffer from NAFLD. ${ }^{5-14}$

NAFLD includes a wide spectrum of liver damage ranging from simple, uncomplicated steatosis to steatohepatitis to advanced fibrosis and cirrhosis. ${ }^{15} 16$ Several studies on the long-term prognosis in the adult population demonstrate that simple steatosis follows a relatively benign clinical course $e^{17} 18$ whereas steatohepatitis associated with increased fibrosis may progress to end-stage liver disease and its resulting complications. $^{18} 19$

Data on prognosis of NAFLD in children remain scant. Some series have reported well-documented cases of cirrhotic stage disease in children ${ }^{120}$ and other series have reported cases of children with NAFLD who developed cirrhosis in young adulthood. ${ }^{21}{ }^{22}$ However, the natural history and prognosis of NAFLD in children remains unknown. Studies of children with NAFLD who underwent long-term follow-up are necessary to better determine the natural history and long-term prognosis of NAFLD in the paediatric population. Thus, we conducted this cohort study aimed at determining the long-term prognosis of children with NAFLD and compare their survival with expected survival of the general population of the United States of the same age and sex.

\section{MATERIAL AND METHODS}

\section{Study design and patient population}

This was a retrospective longitudinal hospitalbased cohort study. The study was approved by the Mayo Institutional Review Board and all patients or responsible guardian gave written informed consent for participation in medical research. Paediatric patients with NAFLD were identified using our Mayo computerised master diagnosis index which is a database of medical records of every patient seen at Mayo Clinic. Each unit medical record contains all inpatient and outpatient medical information for each patient seen at Mayo Clinic since 1907. This has led to the creation of a unified medical index system, the Rochester Epidemiology Project (REP), by which the details of the medical care provided to Mayo patients can be studied. ${ }^{23}$ The REP Mayo computerised master diagnosis indexes all medical diagnoses made at each health encounter by healthcare providers at Mayo. ${ }^{23}$ All diagnoses made in the outpatient office or during clinic visits, hospitalisations, emergency room visits, nursing home care, surgical procedures, autopsies and on death certificates are recorded in the database. Thus, the REP Mayo diagnosis index makes it possible to identify a group of patients with certain characteristics and follow them longitudinally assessing long-term outcomes such as mortality and causes of death.

Patients with a diagnosis of NAFLD were identified by searching the REP master diagnostic 
index using Hospital Adaptation of International Classification of Diseases (HICDA) codes for fatty liver, hepatic steatosis or steatohepatitis (5710-42-42, 5710-43-1, 5710-43-0, 5470-42-0 to 4, 2790-44-1). For the purpose of this study we used the definition of child as an individual under the age of 21 years as proposed by the National Institutes of Health (http://grants. nih.gov/grants/funding/children/children.htm; accessed 13 August 2009). Patients had their first medical evaluation for their liver disease at our institution during a 15 year period from 1 January 1985 to 31 December 1999. The date 1 January 1985 was chosen since the first case of paediatric NAFLD was reported in the mid $1980 \mathrm{~s} ;{ }^{20}$ the date 31 December 1999 was chosen to have a 15 year ascertainment period and a follow-up of more than 5 years for the last patient enrolled. The diagnosis of NAFLD required (1) confirmation of diffuse fatty infiltration of the liver in imaging studies regardless of aminotransferase levels; (2) average daily ethanol consumption of less than $10 \mathrm{~g}$; and (3) appropriate exclusion of other liver diseases based on standard clinical, laboratory, imaging and/or liver biopsy features. Laboratory tests to rule out other liver diseases included a viral hepatitis panel (for hepatitis A, B and C performed either at the time of first evaluation or during the follow-up), ceruloplasmin levels, $\alpha 1$-antitrypsin levels and phenotype, autoantibodies (nuclear antibody (ANA), smooth muscle antibody (SMA), antibody to the liver/kidney microsome type 1, and anti-mitochondrial antibody), and a standard metabolic/inborn error panel (lactate/pyruvate ratio, urine and serum organic acids and amino acids).

The REP master diagnostic index identified a total of 130 cases. After an extensive review of the medical records of these patients, a total of 66 children with unequivocal NAFLD as defined by our diagnostic criteria detailed above were identified. A complete medical history and physical examination, and a complete laboratory evaluation were performed in all patients at the time of first medical evaluation in our institution and repeated at regular intervals thereafter. Laboratory evaluation included liver biochemistries (serum aspartate aminotransferase (AST), alanine aminotransferase (ALT), alkaline phosphatase activity, $\gamma$-glutamyl transferase (GGT), total bilirubin, albumin levels and prothrombin time), fasting blood glucose, fasting lipid profiles (triglyceride, total cholesterol, HDL-cholesterol and LDL-cholesterol levels), and specific laboratory tests to rule other liver diseases as described before. All patients underwent abdominal imaging with ultrasonography, computed tomography scan, and/or magnetic resonance imaging confirming the presence of fatty infiltration of the liver.

The body mass index (BMI) based on body weight (kilograms) divided by the square of height (metres) was calculated in every case. BMI percentile was determined according to age and sex based on data from the Center for Disease Control and Prevention. ${ }^{24}$ Obesity was defined by a BMI $>95^{\text {th }}$ percentile for age and sex. ${ }^{24}$ Abnormalities in the fasting levels of triglycerides and HDL-cholesterol were adjusted according to age, sex and race or ethnic group ( $>95^{\text {th }}$ percentile for triglycerides; $<5^{\text {th }}$ percentile for HDL-cholesterol) as recommended. ${ }^{25}$ Diabetes mellitus was diagnosed based on standard criteria as recommended by the American Diabetes Association. ${ }^{26}$ Hypertension was defined as a systolic or diastolic value that exceeded the $95^{\text {th }}$ percentile for age, sex and height. ${ }^{27}$ Hypercholesterolaemia was defined as a fasting total cholesterol level $\geqslant 200 \mathrm{mg} / \mathrm{dl} .{ }^{28} \mathrm{High}$ LDL-cholesterol was defined by a LDL-cholesterol level $\geqslant 130 \mathrm{mg} / \mathrm{dl}^{28}$

In addition, patients were classified as having the metabolic syndrome if they met three or more of the following five criteria for age and sex as proposed: ${ }^{29}$ BMI above the $97^{\text {th }}$ percentile (which corresponds to a $z$-score of 2.0 or more); triglyceride level above the $95^{\text {th }}$ percentile; HDL cholesterol level below the $5^{\text {th }}$ percentile; systolic or diastolic blood pressure above the $95^{\text {th }}$ percentile; and impaired glucose tolerance. As an oral glucose tolerance test was not performed, we used a fasting glucose value of at least $100 \mathrm{mg} / \mathrm{dl}$ to replace impaired glucose tolerance, as recently proposed by the International Diabetes Federation. ${ }^{30}$

\section{Liver histology}

A baseline liver biopsy was performed in 29 patients at the time of diagnosis and follow-up liver biopsies in five of these patients. Since there are no established guidelines of when to perform a liver biopsy in patients with NAFLD, the decision to perform a baseline liver biopsy in our patient population was made on an individual basis by the treating gastroenterologist, and in most $(82 \%)$ cases was performed due to persistently abnormal liver enzymes. Liver biopsy features including grade of steatosis, inflammatory infiltrate, and ballooning, the presence of Mallory hyaline, and stage of fibrosis were graded according to the scoring system proposed by Kleiner et al. ${ }^{31}$

\section{Statistical analysis}

Continuous variables are presented as mean with the standard deviation (SD), and discrete variables are expressed as the number (percentage) of patients with a condition. Comparisons between patients with and without liver biopsy were performed with two-sample t tests for continuous variables and $\chi^{2}$ tests for categorical variables. Survival curves were created using the Kaplan-Meier method. The starting point for survival analysis was date of diagnosis of NAFLD. Patient follow-up was extended up to April 2008. The end-points for survival analysis were death or liver transplantation. For survival comparison we calculated the expected number of deaths for a cohort with the same age and sex distribution and the same amount of observation time (exposure to death) as the 66 children with NAFLD. The estimates were made using mortality data for United States from the U.S. Center for Health Statistics as previously detailed. ${ }^{32}$ We used the relationship between the logrank test and the Poisson distribution. The $\mathrm{p}$ value calculated (from the one-sample log-rank test) depends on the assumption that the number of deaths follows a Poisson distribution with an expected value equal to the expected number of deaths. ${ }^{32}$ The standardised mortality ratio (SMR) was calculated using the Ederer method based on age and sex to derive the expected number of events. ${ }^{33}$

\section{RESULTS}

\section{Clinical features at presentation}

The main demographic and clinical features are summarised in table 1. There was a slightly higher proportion of boys than girls, and two-thirds were obese. Most patients had symptoms or signs at presentation. The features of the metabolic syndrome were common with more than half having a BMI $>97^{\text {th }}$ percentile. Fifty-five $(83.3 \%)$ children presented with at least one feature of the metabolic syndrome whereas overt metabolic syndrome (ie, $\geqslant 3$ features) was present in 19 (28.8\%) children. Other features that worsen the cardiovascular risk profile such as hypercholesterolaemia and high LDL-cholesterol were also common. The main laboratory data gathered at the time of NAFLD diagnosis are summarised in table 2. ALT and AST levels were each within the normal range in few patients. 
Table 1 Demographic and clinical features at presentation $(n=66)$

\begin{tabular}{ll}
\hline Variable & Mean (SD) or number (\%) \\
\hline Age (years) & $13.9(\mathrm{SD} 3.9)$ (range, 3.2-19.6) \\
$\geqslant 10$ years & $55(83.3 \%)$ \\
Gender (M/F) & $37 / 29$ \\
BMI $\left(\mathrm{kg} / \mathrm{m}^{2}\right)$ & $31.2(\mathrm{SD} 7.6)$
\end{tabular}

Type of presentation Asymptomatic

$17(25.8 \%)$

Symptomatic

$49(74.2 \%)$

$\begin{array}{lc}\text { Signs and symptoms* } & \\ \text { Abdominal pain } & 31(47 \%) \\ \text { Fatigue } & 19(28.8 \%) \\ \text { Hepatomegaly } & 18(27.3 \%) \\ \text { Splenomegaly } & 3(4.5 \%) \\ \text { Acanthosis nigricans } & 5(7.6 \%)\end{array}$

\section{Associated conditions ${ }^{\dagger}$}

Obesity (BMI $>95^{\text {th }}$ percentile) $42(65.6 \%)$

$\mathrm{BMI}>97^{\text {th }}$ percentile $(z$-score, $\quad 38(57.6 \%)$

2.0 or more)

Hypertriglyceridaemia

$31(47.0 \%)$

$18(27.3 \%)$

$17(25.8 \%)$

$8(12.1 \%)$

$27(40.9 \%)$

$15(22.7 \%)$

High LDL-cholesterol

$\begin{array}{ll}\begin{array}{l}\text { Features of metabolic } \\ \text { syndrome: }\end{array} & \\ 0 & 11(16.7 \%) \\ 1 \text { or more } & 55(83.3 \%) \\ 2 \text { or more } & 48(72.7 \%) \\ 3 \text { or more } & 19(28.8 \%)\end{array}$

*Some patients presented with more than one symptom or associated condition.

+ Hypertriglyceridaemia was defined as a level above the $95^{\text {th }}$ percentile for age and sex; low HDL-cholesterol means a level below the $5^{\text {th }}$ percentile for age and sex; hypertension means a systolic or diastolic blood pressure above the $95^{\text {th }}$ percentile for age, sex and height; hypercholesterolaemia means a level $\geqslant 200 \mathrm{mg} / \mathrm{dl}$; hyperglycaemia means a level of $\geqslant 100 \mathrm{mg} / \mathrm{dl}$; and high LDLcholesterol means a level $\geqslant 130 \mathrm{mg} / \mathrm{dl}$.

\$The metabolic syndrome was diagnosed in patients who met three or more of the following criteria for age and sex: a BMl above the $97^{\text {th }}$ percentile ( $z$-score, 2.0 or more), a triglyceride level above the $95^{\text {th }}$ percentile, an HDL-cholesterol level below the $5^{\text {th }}$ percentile systolic or diastolic blood pressure above the $95^{\text {th }}$ percentile, and a fasting glucose value of at least $100 \mathrm{mg} / \mathrm{dl}$ as proposed. ${ }^{29}{ }^{30}$ BMI, body mass index; F, female; HDL, high-density lipoprotein; LDL, low-density lipoprotein; M, male.

The AST/ALT ratio was greater than 1 in $29 \%$ of children. GGT was elevated in $88 \%$ of patients while serum alkaline phosphatase was above the normal value for age and sex in few patients. Serum total bilirubin, albumin and prothrombin time were essentially within the normal range in all patients. Positive autoantibodies in low titres were found in $20 \%$ of patients including ANA in $15.4 \%$, and SMA in $10 \%$ of patients.

\section{Liver histology}

The main liver biopsy features are summarised in table 3. Some degree of fibrosis was present in $59 \%$ of children including mild fibrosis (stage 1-2) in 11, septal/bridging fibrosis (stage 3 ) in four, and cirrhotic-stage disease in two. The mean NAFLD activity score was 3.5 (SD 1.02). Portal based injury was seen in nine $(31 \%)$ children but associated with zone 3 injury in most. Interface hepatitis or other features suggestive of autoimmune hepatitis were not present in any case. As summarised in table 4, patients undergoing liver biopsy had significantly higher levels of ALT and lower levels of total cholesterol and triglycerides; otherwise patients undergoing liver biopsy were similar to those who were not biopsied.

\section{Long-term follow-up}

The mean follow-up of the total cohort was 6.4 (SD 4.5) (range, 0.05-20) years, for a total of 409.6 person-years. During this time, treatment recommendations included lifestyle modifications consisting of an exercise programme along with diet modifications tailored to individual need and preference alone or in eight (12.1\%) patients in combination with either ursodeoxycholic acid or vitamin E. One year after initiation of the prescribed lifestyle modification, $49 \%$ of children were able to lose at least $10 \%$ of their baseline weight, and $86 \%$ of them showed significant improvement or normalisation of aminostransferases. Neither ursodeoxycholic acid nor vitamin $\mathrm{E}$ treatment appeared to impact further the liver enzymes levels although their effect independent of weight loss could not be assessed due to the very small number of patients on either treatment. At the time of last follow-up, however, most patients (76\%) had re-gained weight, and in $46 \%$ of them aminotransferases returned to baseline values. Interestingly, four children developed type 2 diabetes 4, 6, 7 and 11 years after the diagnosis of NAFLD. Other complications that occurred during follow-up were cholecystitis requiring cholecystectomy (six patients), morbid obesity requiring bariatric surgery (two patients), contraceptive-induced liver injury (one patient) and bilateral oophorectomy and hysterectomy for endometriosis (one patient).

A total of 13 liver biopsies were obtained from five patients over a mean period of 41.4 (SD 28.8) months (table 5). The grade of steatosis and lobular inflammation either worsened or remained the same in all patients. Progression of fibrosis stage was documented in four cases. One patient without fibrosis at presentation developed stage 1 fibrosis at 19 months, and cirrhosis (stage 4 fibrosis) at 57 months. Another patient presented without fibrosis, but progressed to stage 1 fibrosis at 39 months, and to stage 3 fibrosis at 82 months. Two other patients without fibrosis at presentation progressed to stage 1 fibrosis, one at 28 months and the other at 7 months. There was no improvement in any of those histological features in any case.

\section{Long-term survival}

During follow-up, two patients underwent liver transplantation, and two additional patients died. The observed number of events in the total person-years of follow up was $4 / 409.6=9.8$ per thousand. The observed number of events in the NAFLD cohort was significantly higher than the expected number of events in the United States population of same age and sex (4 vs $0.29416, p<0.00001)$ with a SMR of 13.6 (95\% confidence intervals, 3.8 to 34.8). The observed survival free of liver transplantation in the NAFLD cohort as compared to the expected survival of the general United States population of the same age and sex is illustrated in fig 1.

The two patients who underwent liver transplantation were those two who presented with cirrhosis on liver biopsy. The first case was a Hispanic female diagnosed with cirrhotic-stage non-alcoholic steatohepatitis (NASH) at 11 years of age, when she presented with a BMI of $26.9 \mathrm{~kg} / \mathrm{m}^{2}$, hypercholesterolaemia and hypertriglyceridaemia. She was found with grade 3 
Table 2 Laboratory features at presentation $(n=66)$

\begin{tabular}{|c|c|c|c|c|}
\hline Laboratory test & Mean (SD) or proportion & Range & 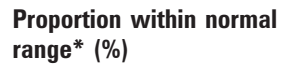 & Normal range $\dagger$ \\
\hline Alanine aminotransferase (U/l) & $137(102)$ & $17-579$ & 6 & $10-45$ \\
\hline Serum aspartate aminotransferase (U/l) & $94(62)$ & $18-326$ & 14 & $26-31$ \\
\hline$\gamma$-Glutamytransferase (U/I) & $77(52)$ & $25-210$ & 12 & $6-31$ \\
\hline Total bilirubin (mg/dl) & $0.7(0.6)$ & $0.2-4.3$ & 90 & $0.1-1.0$ \\
\hline Albumin (g/dl) & $4.5(0.4)$ & $3.6-5.2$ & 100 & $3.5-5.0$ \\
\hline HDL-cholesterol & $37(11.5)$ & $11-67$ & 72.7 & $t$ \\
\hline LDL-cholesterol & $130(49)$ & $55-233$ & 77.3 & $<130$ \\
\hline Triglyceride (mg/dl) & $216(168)$ & $44-768$ & 53 & $\dagger$ \\
\hline Glucose (mg/dl) & $106(51)$ & 73-371 & 87.9 & $<100$ \\
\hline Anti-nuclear antibodies & $15.4 \%$ & & & Negative \\
\hline Smooth muscle antibodies & $10 \%$ & & & Negative \\
\hline
\end{tabular}

* Refers to patients who had normal laboratory values considering the normal range for the specific age and sex in each individual case. HIncludes the normal laboratory values for boys and girls for the age range of our patient population.

:Based on percentile for age and sex.

ALT, alanine aminotransferase; ANA, antinuclear antibody; AST, serum aspartate aminotransferase; GGT, $\gamma$-glutamyl transferase; HDL, high-density lipoprotein; LDL, low-density lipoprotein; SAM, smooth muscle antibody.

oesophageal varices and developed recurrent variceal bleeding requiring variceal band ligation in multiple occasions. She underwent liver transplantation at 20 years of age due to endstage liver disease and hepatopulmonary syndrome. In the posttransplant period, she was diagnosed with recurrent NASH at 9 months, stage 1 fibrosis at 2 years and 3 months, and stage 2 fibrosis at 3 years and 3 months after liver transplantation. She

Table 3 Liver biopsy features $(n=29)$

\begin{tabular}{lc}
\hline Histological finding & Number (\%) \\
\hline Steatosis score & \\
0, Minimal $(<5 \%)$ & $5(17.2)$ \\
1, Mild $(>5-33 \%)$ & $11(37.9)$ \\
2, Moderate $(>33 \%$ and $66 \%)$ & $13(44.8)$ \\
3, Severe $>66 \%)$ & \\
Lobular inflammation score & $1(3.4)$ \\
0, No foci & $26(89.7)$ \\
1, Mild & $1(3.4)$ \\
2, Moderate & $1(3.4)$ \\
3, Severe & \\
Hepatocellular ballooning & $24(82.8)$ \\
0, None & $5(17.2)$ \\
1, Few & 0 \\
2, Prominent & \\
NAFLD activity score & $5(17.2)$ \\
1-2 & $20(70)$ \\
3-4 & $4(13.8)$ \\
5 or more & \\
Fibrosis & $12(41.3)$ \\
Stage 0 & \\
Stage 1 Perisinusoidal or periportal & $3(10.3)$ \\
1A Delicate perisinusoidal & $1(3.5)$ \\
1B Dense perisinusoidal & $2(6.9)$ \\
1C Periportal only & $5(17.2)$ \\
Stage 2 Perisinusoidal and periportal & $4(13.8)$ \\
Stage 3 Bridging fibrosis & $2(6.9)$ \\
Stage 4 Cirrhosis & $4(13.8)$ \\
Mallory's hyaline & \\
\hline
\end{tabular}

Liver biopsy features were graded and staged according to the scoring system proposed by Kleiner et al. ${ }^{31}$ The grade of steatosis $(0-3)$, lobular inflammation $(0-3)$, and ballooning $(0-2)$ were then combined to determine the non-alcoholic fatty liver disease (NAFLD) activity score $(0-8)$ as proposed. ${ }^{31}$ is currently alive. The second case was a white female diagnosed with cirrhotic-stage NASH at 18.9 years of age when presented with a BMI of $33.6 \mathrm{~kg} / \mathrm{m}^{2}$, and low HDL-cholesterol. She developed severe hypoxaemia from hepatopulmonary syndrome without any other liver complication requiring liver transplantation at 25 years of age. She was found with macrovesicular steatosis on protocol liver biopsy as early as 14 days after liver transplantation, with well-established NASH at 6 weeks after liver transplantation, and with bridging fibrosis at 1 year. She was diagnosed with cirrhotic stage NASH in the graft and hepatopulmonary syndrome 2 years after liver transplantation, requiring re-transplantation 2.3 years after the first liver transplant procedure. Finally, she died from multiple organ failure at age 27 years. The two deaths recorded were both nonliver related, and none of these two cases had liver biopsy performed at any time.

\section{DISCUSSION}

The study is the first to describe the long-term survival of children with NAFLD who underwent a follow-up of up to 20 years. The study demonstrates that NAFLD in children is a disease of progressive potential. Some children presented with cirrhosis, others progressed to advanced fibrosis or cirrhosis during follow-up, and some developed end-stage liver disease with the consequent need of liver transplantation. The study shows that NAFLD in children is associated with a significantly shorter long-term survival as compared to the expected survival of the general population of the same age and sex; our children with NAFLD had a 13.8-fold higher risk of dying or requiring liver transplantation than the general population of the same age and sex. The two deaths recorded were not liver related, but the inclusion of these two cases among the four cases reaching the outcome of death or liver transplantation is appropriate as the comparison was done to overall mortality in the general population of same age and sex regardless of the causes of death.

The study also provides interesting data regarding the progressive potential of NAFLD to more advanced disease. Four of the five children with repeated liver biopsy did not have fibrosis on diagnosis liver biopsy, but two developed mild (stage 1) fibrosis, and the other two developed advanced (stage 3-4) fibrosis. The progression of liver damage in these patients over a relatively short period of time highlights the importance of 
Table 4 Comparison of major variables between patients with or without liver biopsy

\begin{tabular}{|c|c|c|c|}
\hline Variable & $\begin{array}{l}\text { Liver biopsy }(n=29) \text {; } \\
\text { mean }(S D) \text { or } n(\%)\end{array}$ & $\begin{array}{l}\text { No liver biopsy }(n=37) \text {; } \\
\text { mean (SD) or } n(\%)\end{array}$ & p Value \\
\hline Age (years) & $13.2(4.1)$ & $14.6(3.7)$ & 0.15 \\
\hline Male gender (male/total) & $19(65 \%)$ & $18(49 \%)$ & 0.17 \\
\hline BMI $\left(\mathrm{kg} / \mathrm{m}^{2}\right)$ & $30.1(6.5)$ & $32.1(8.3)$ & 0.29 \\
\hline Abdominal pain at presentation & $13(45 \%)$ & $18(48 \%)$ & 0.76 \\
\hline Obesity (BMI $>95^{\text {th }}$ percentile) & $18(55 \%)$ & $24(65 \%)$ & 0.96 \\
\hline Dyslipidaemia & $10(34 \%)$ & $14(38 \%)$ & 0.78 \\
\hline Hypertriglyceridaemia & $12(41 \%)$ & $19(51 \%)$ & 0.47 \\
\hline Hypercholesterolaemia & $12(41 \%)$ & $14(38 \%)$ & 0.89 \\
\hline Hypertension & $3(10 \%)$ & $5(13 \%)$ & 0.69 \\
\hline ALT (IU/I) & $166.7(125.8)$ & $105.1(54.9)$ & 0.03 \\
\hline AST (IU/I) & $103.3(69.4)$ & $87.0(54.6)$ & 0.29 \\
\hline Glucose (mg/dl) & $92.9(13.0)$ & $117.3(66.8)$ & 0.09 \\
\hline Cholesterol (mg/dl) & $193.6(41.6)$ & $235.4(92.6)$ & 0.04 \\
\hline Triglyceride (mg/dl) & $149.9(61.1)$ & $272.1(208.1)$ & 0.007 \\
\hline Duration of follow-up (years) & $6.2(4.7)$ & $6.6(4.2)$ & 0.73 \\
\hline
\end{tabular}

ALT, alanine aminotransferase; AST, serum aspartate aminotransferase; BMI, body mass index.

identifying those children with NAFLD who are at risk of having a more progressive liver disease. In some recent series, the presence and severity of fibrosis was consistently associated with a higher BMI or larger waist circumference. ${ }^{10}{ }^{12}{ }^{14}$ Older age and higher levels of AST and insulin have been found associated with fibrosis in some series ${ }^{10}{ }^{12}$ However, further studies are needed to accurately identify those children who are more likely to progress to end-stage liver disease.

Interestingly, the two patients in our cohort who underwent liver transplantation had hepatopulmonary syndrome as the main indication for transplant. However, whether or not there is an association between progression to cirrhosis and development of severe hepatopulmonary syndrome requiring liver transplantation in paediatric NAFLD remains uncertain, and further studies in this area are needed. It is also intriguing that both cases undergoing liver transplantation in our series developed recurrent NASH, with cirrhotic stage disease in one patient who required re-transplantation. Recurrence of NASH after liver transplantation in children has been documented in two isolated cases, ${ }^{34}{ }^{35}$ both male patients of age 13 and 16 years who developed decompensated liver disease from NAFLD. Both patients had a history of hypothalamic/pituitary dysfunction; in one case associated with hepatopulmonary syndrome. These two cases $^{34} 35$ extended prior observations of the development of severe liver disease from NAFLD in patients with hypothalamic/ pituitary dysfunction. ${ }^{36}$

Similar to other paediatric series of NAFLD, most of our children were diagnosed in the second decade of life; girls and boys were affected almost equally with a slight male predominance, and most were symptomatic at presentation.

As in adults with NAFLD, a high proportion of our children were obese and had several features associated with the metabolic syndrome. Unlike adults, almost a third of our children had portal-based injury on liver biopsy, but most of them had pericentral/perisinusoidal injury as well. Two of our children had type 2 diabetes prior to the diagnosis of NAFLD, whereas four patients developed type 2 diabetes within 11 years after NAFLD was diagnosed. Therefore, children with NAFLD

Table 5 Follow-up liver biopsy $(n=5)$

\begin{tabular}{|c|c|c|c|c|c|c|}
\hline Patient no & $\begin{array}{l}\text { Interval between first and last } \\
\text { liver biopsy (months) }\end{array}$ & $\begin{array}{l}\text { Date of liver biopsy } \\
\text { (month/date/year) }\end{array}$ & Steatosis grade & $\begin{array}{l}\text { Lobular } \\
\text { inflammation grade }\end{array}$ & Fibrosis stage & Reason to repeat the liver biopsy \\
\hline \multirow[t]{2}{*}{1} & \multirow[t]{2}{*}{33} & First biopsy (3/1993) & Moderate & Mild & 1 & - \\
\hline & & Second biopsy (12/1995) & Moderate & Mild & 1 & $\begin{array}{l}\text { Follow-up before ursodeoxycholic acid } \\
\text { prescribed }\end{array}$ \\
\hline \multirow[t]{3}{*}{2} & \multirow[t]{3}{*}{57} & First biopsy (7/1999) & Mild & Mild & 0 & - \\
\hline & & Second biopsy $(2 / 2001)$ & Moderate & Mild & 1 & Worsening of ALT levels \\
\hline & & Last biopsy (4/2004) & Severe & Mild & $\underline{4}$ & $\begin{array}{l}\text { Liver biopsy performed for persistently } \\
\text { elevated ALT and done during } \\
\text { laparoscopic cholecystectomy }\end{array}$ \\
\hline \multirow[t]{3}{*}{3} & \multirow[t]{3}{*}{82} & First biopsy $(12 / 1996)$ & Mild & Mild & 0 & - \\
\hline & & Second biopsy (4/1999) & Moderate & Mild & 1 & Persistent abnormal liver tests \\
\hline & & Last biopsy (11/2002) & Moderate & Moderate & 3 & $\begin{array}{l}\text { Liver biopsy performed for persistently } \\
\text { elevated liver enzymes and done during } \\
\text { bariatric surgery }\end{array}$ \\
\hline \multirow[t]{3}{*}{4} & \multirow[t]{3}{*}{28} & First biopsy (8/1990) & Moderate & Mild & 0 & - \\
\hline & & Second biopsy $(2 / 1991)$ & Moderate & Mild & 0 & Persistent abnormal liver tests \\
\hline & & Third biopsy (12/1992) & Moderate & Mild & 1 & $\begin{array}{l}\text { Follow-up before starting treatment with } \\
\text { ursodeoxycholic acid }\end{array}$ \\
\hline \multirow[t]{2}{*}{5} & \multirow[t]{2}{*}{7} & First biopsy (14 0ct 1998) & Mild & 0 & 0 & - \\
\hline & & Second biopsy (10/1999) & Mild & 0 & 1 & $\begin{array}{l}\text { Laparoscopic liver biopsy during surgery } \\
\text { for persistent patent ductus venosis }\end{array}$ \\
\hline
\end{tabular}




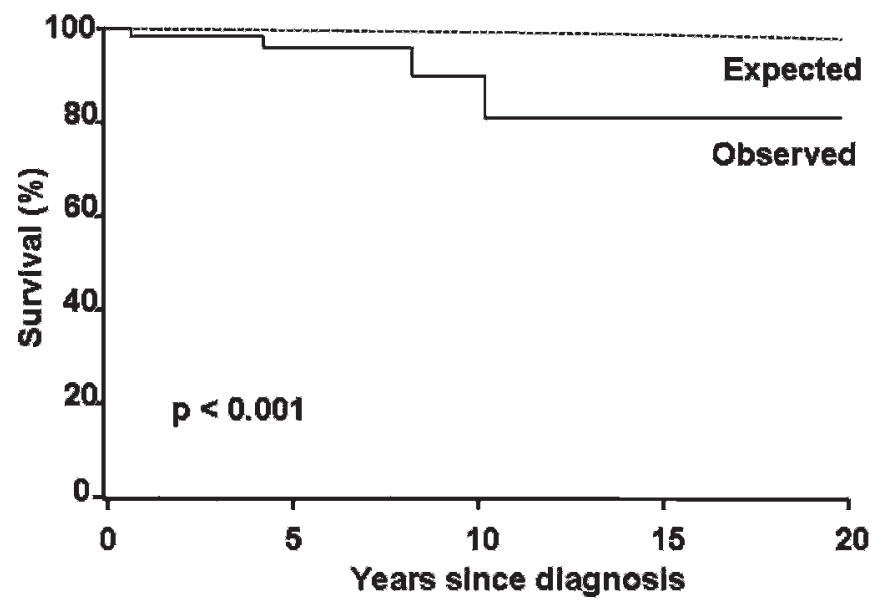

Figure 1 Kaplan-Meier survival curve of children with non-alcoholic fatty liver disease (NAFLD) $(n=66)$ as compared to the general United States population of same age and sex. The observed survival free of liver transplantation was significantly shorter in the NAFLD cohort as compared to the expected survival of the general United States population of the same age and sex; the standarised mortality ratio was 13.6 (95\% confidence intervals, 3.8 to $34.8 ; p<0.0001$ ).

should be closely monitored for development of type 2 diabetes later in life.

We found a high proportion of children (27.3\%) with HDLcholesterol below the $5^{\text {th }}$ percentile for their age and sex which has not been reported in paediatric NAFLD before. Similar to adults with NAFLD, ${ }^{37} 20 \%$ of our children tested positive for low titre of ANA and/or SMA. Interestingly, the vast majority $(88 \%)$ of our children had elevated GGT with alkaline phosphatase levels within the normal range in most of them. To our knowledge, this high proportion of children with elevated GGT levels has not been described in any other series of paediatric NAFLD. Recently, higher serum levels of GGT have been associated with several cardiovascular disease risk factors or components of the metabolic syndrome. ${ }^{38-41}$ GGT is located on the external surface of most cells and mediates the uptake of glutathione, an important component of intracellular antioxidant defences. GGT could be informative in children with NAFLD because its expression is enhanced by oxidative stress and it could be released by several conditions inducing cellular stress and insulin resistance; both insulin resistance and oxidative stress are key components in the development of NAFLD. ${ }^{42}$

The main strengths of our study are the inclusion of children with the whole spectrum of NAFLD from simple steatosis to cirrhosis along with the long-term follow-up of up to 20 years. The cases were well documented with all children having the diagnosis of NAFLD confirmed by radiological findings, and in almost half of them with liver histology. However, our study has some limitations. First, our patients were seen in a referral tertiary care medical centre, and although the results may be extrapolated to other similar medical centres, the results most likely may not apply to children with NAFLD from the community. In this regard, larger community- or populationbased studies are necessary to determine the prognosis of NAFLD in children from the general population. Second, most of our children $(80 \%)$ were white, and thus, whether or not the long-term prognosis of paediatric NAFLD is any different among the different ethnic groups needs to be investigated. Finally, since liver biopsy is not part of the standard of care to confirm the diagnosis of NAFLD, only about a half of our children underwent liver biopsy and, thus, we were not able to determine the prognostic significance of the individual histological features.

In summary, our study demonstrates that NAFLD in children is associated with a significantly shorter survival as compared to survival of the general population of same age and sex. NAFLD in children may progress to cirrhosis and end-stage liver disease with the consequent need for liver transplantation, but NAFLD with severe NASH may recur in the allograft. Further studies are needed to identify those children with NAFLD who are at a higher risk for disease progression who would be expected to benefit the most from medical therapy.

Funding: PC was supported by a grant from the Faculty of Medicine, Siriraj Hospital, Mahidol University, Bangkok, Thailand. ST was supported by a medical research scholarship from the Faculty of Medicine, Chulalongkorn University, Bangkok, Thailand.

Competing interests: None.

Ethics approval: The study was approved by the Mayo Institutional Review Board on 18 June 2002.

Provenance and peer review: Not commissioned; externally peer reviewed

\section{REFERENCES}

1. Schwimmer JB, Deutsch $R$, Kahen $T$, et al. Prevalence of fatty liver in children and adolescents. Pediatrics 2006;118:1388-93.

2. Tominaga K, Kurata JH, Chen YK, et al. prevalence of fatty liver in Japanese children and relationship to obesity. An epidemiological ultrasonographic survey. Dig Dis Sci 1995; 40:2002-9.

3. Franzese A, Vajro P, Argenziano A, et al. Liver involvement in obese children. Ultrasonography and liver enzymes levels at diagnosis and during follow-up in an Italian population. Dig Dis Sci 1997;42:1428-32.

4. Chan DF, Li AM, Chu WC, et al. Hepatic steatosis in obese Chinese children. Int J Obes Relat Metab Disord 2004;28:1257-63.

5. Baldridge AD, Perez-Atayde AR, Grame-Cooke F, et al. Idiopathic steatohepatitis in childhood: A multicenter retrospective study. J Pediatr 1995;127:700-4.

6. Franzese A, Vajro $P$, Argenziano $A$, et al. Liver involvement in obese children. Ultrasonography and liver enzyme levels at diagnosis and during follow-up in an Italian population. Dig Dis Sci 1997;42:1428-32.

7. Rashid M, Roberts EA. Nonalcoholic steatohepatitis in children. J Pediatric Gastroenterol Nutr 2000;30:48-53.

8. Demir H, Kocak N, Gurakan F, et al. Obesity: a cause of steatohepatitis in children. Am J Gastroenterol 2000;95:1366-7.

9. Manton ND, Lipsett J, Moore DJ, et al. Nonalcoholic steatohepatitis in children and adolescents. Med J Aust 2000;173:476-9.

10. Schwimmer JB, Deutsch R, Rauch JB, et al. Obesity, insulin resistance, and othe clinicopathological correlates of pediatric nonalcoholic fatty liver disease. J Pediatr 2003;143:500-5.

11. Schwimmer JB, McGreal N, Deutsch R, et al. Influence of gender, race, and ethnicity on suspected fatty liver in obese adolescents. Pediatrics 2005;115:e561-5.

12. Nobili V, Marcellini M, Devito $R$, et al. NAFLD in children: a prospective clinicalpathological study and effect of lifestyle advice. Hepatology 2006;44:458-65.

13. Patton HM, Sirlin C, Behling C, et al. Pediatric nonalcoholic fatty liver disease: a critical appraisal of current data and implications for future research. J Pediatr Gastroenterol Nutr 2006;43:413-27.

14. Manco M, Marcellini M, Devito R, et al. Metabolic syndrome and liver histology in paediatric non-alcoholic steatohepatitis. Int J Obes (Lond) 2008;32:381-7.

15. Angulo P. Nonalcoholic fatty liver disease. N Engl J Med 2002;346:1221-31.

16. Brunt EM, Janney CG, Di Bisceglie AM, et al. Nonalcoholic steatohepatitis: a proposal for grading and staging the histological lesions. Am J Gastroenterol 1999;94:2467-74.

17. Dam-Larsen S, Franzmann M, Andersen IB, et al. Long term prognosis of fatty liver: risk of chronic liver disease and death. Gut 2004;53:750-5.

18. Ekstedt M, Franzen LE, Mathiensen Ul, et al. Long-term follow-up of patients with NAFLD and elevated liver enzymes. Hepatology 2006;44:865-73.

19. Adams LA, Lymp JF, St Sauver J, et al. The natural history of nonalcoholic fatty liver disease: a population-based cohort study. Gastroenterology 2005;129:113-21.

20. Kinugasa A, Tsunamoto K, Furukawa N, et al. Fatty liver and its fibrous changes found in simple obesity of children. J Pediatr Gatroenterol Nutr 1984;3:408-14.

21. Molleston JP, White F, Teckman J, et al. Obese children with steatohepatitis can develop cirrhosis in childhood. Am J Gastroenterol 2002;97:2460-2.

22. Suzuki D, Hashimoto E, Kaneda K, et al. Liver failure caused by non-alcoholic steatohepatitis in an obese young male. J Gastroenterol Hepatol 2005;20:327-9.

23. Melton LJ 3rd. History of the Rochester Epidemiology Project. Mayo Clin Proc 1996;71:266-74.

24. Kuczmarski RJ, Ogden CL, Grummer-Strawn LM, et al. CDC growth charts: United States. Adv Data 2000;8:1-27. 
25. NGHS Coordinating Center. NHLBI Growth and Health Study (NGHS) data monitoring report. Baltimore: Maryland Medical Research, 1998.

26. American Diabetes Association. Diagnosis and Classification of Diabetes Mellitus. Diabetes Care 2005:28(Suppl 1):S37-S42.

27. National High Blood Pressure Education Program. Update on the 1987 Task Force Report on High Blood Pressure in Children and Adolescents: a working group report from the National High Blood Pressure Education Program. Pediatrics 1996;98:649-58.

28. National Cholesterol Education Program. Report of the Expert Panel on Blood Cholesterol in Children and Adolescents. Pediatrics 1998;101:141-7.

29. Weiss R, Dziura J, Burgert TS, et al. Obesity and the metabolic syndrome in children and adolescents. N Engl J Med 2004;350:2362-74.

30. Zimmet $\mathbf{P}$, Alberti G, Kaufman F, et al. The metabolic syndrome in children and adolescents. Lancet 2007:369:2059-61.

31. Kleiner DE, Brunt EM, Van Natta M, et al. Design and validation of a histological scoring system for nonalcoholic fatty liver disease. Hepatology 2005; 41:1313-21.

32. Therneau T, Offord J. Expected survival based on hard rates (update). Rochester MN: Department of Health Science Research, Section of Biostatistics, Mayo Clinic 1999, Technical Report Series No. 63.

33. Ederer F. The relative survival rate: a statistical methodology. National Cancer Institute Monographs 1961;6:101-21.
34. Jonas MM, Krawczuk LE, Kim HB, et al. Rapid recurrence of nonalcoholic fatty liver disease after transplantation in a child with hypopituitarism and hepatopulmonary syndrome. Liver Transp/ 2005;11:108-10.

35. Jankowska I, Socha P, Pawlowska J, et al. Recurrence of non-alcoholic steatohepatitis after liver transplantation in a 13-yr-old boy. Pediatr Transplant 2007;11:796-8.

36. Adams LA, Feldstein A, Lindor KD, et al. Nonalcoholic fatty liver disease among patients with hypothalamic and pituitary dysfunction. Hepatology 2004;39:909-14.

37. Adams LA, Lindor KD, Angulo P. The prevalence of autoantibodies and autoimmune hepatitis in patients with nonalcoholic fatty liver disease. Am J Gastroenterol 2004;99:1316-20.

38. Lee DH, Ha MH, Kim JH, et al. Gamma-glutamyltransferase and diabetes-a 4 year follow-up study. Diabetologia 2003;46:359-64.

39. Nakanishi N, Nishina K, Li W, et al. Serum gamma-glutamyltransferase and development of impaired fasting glucose or type 2 diabetes in middle-aged Japanese men. J Intern Med 2003;254:287-95.

40. Kim DJ, Noh JH, Cho NH, et al. Serum gamma-glutamyltransferase within its normal concentration range is related to the presence of diabetes and cardiovascular risk factors. Diabet Med 2005;22:1134-40.

41. Kang YH, Min HK, Son SM, et al. The association of serum gamma glutamyltransferase with components of the metabolic syndrome in the Korean adults. Diabetes Res Clin Pract 2007;77:306-13.

42. Day CP. From fat to inflammation. Gastroenterology 2006;130:207-10.

\title{
Editor's quiz: GI snapshot
}

\begin{abstract}
ANSWER
From the question on page 1466

The CT scan shows a pelvic mass abutting the sigmoid colon causing large bowel obstruction. Histology confirmed the presence of large spherical clusters of actinomycosis colonies. This case demonstrates a rare cause of large bowel obstruction from an ascending actinomycosis infection caused by the IUCD.

Actinomycosis is a chronic, progressive suppurative granulomatous infection caused by a Gram-positive, microaerophillic, filamentous bacterium. ${ }^{1}$ This pathogen can be found as a normal commensal in the gastrointestinal and genital tract; Actinomycosis israelii being the most common subtype. ${ }^{1}$

Actinomycosis classically becomes opportunistic in females with a long-term IUCD and progresses as an ascending genital tract infection affecting both pelvic and adjacent abdominal organs. ${ }^{2}$ It is estimated that actinomycosis is present in $10 \%$ of asymptomatic IUCD users; this rises to $25 \%$ in patients with genitourinary tract infections. ${ }^{2}$

Patients usually present with symptoms often mimicking pelvic malignancy or diverticulitis. Preoperative recognition of the infection is difficult and is found in $<10 \%$ of cases prior to surgery. ${ }^{3}$

Imaging modalities are non-specific, although CT or MRI may show the presence of pelvic soft tissue mass. The mainstay of treatment for actinomycosis remains antibiotics in the form of penicillins and removal of the IUCD. ${ }^{5}$
\end{abstract}

Gut 2009;58:1544. doi:10.1136/gut.2009.178392a

\section{REFERENCES}

1. Valko P, Busolini E, Donati N, et al. Severe large bowel obstruction secondary to infection with Actinomyces israelii. Scand J Infect Dis 2006;38:231-4.

2. Nasu K, Matsumoto H, Yoshimatsu J, et al. Ureteral and sigmoid obstruction caused by pelvic actinomycosis in an intrauterine contraceptive device user. Gynecol Obstet Invest 2002;54:228-31.

3. Chen LW, Chang LC, Shie SS, et al. Solitary actinomycotic abscesses of liver: report of two cases. Int J Clin Pract 2006;60:104-7.

4. Kim JC, Cho MK, Yook JW, et al. Extensive colonic stricture due to pelvic actinomycosis. J Korean Med Sci 1995;10:142-6.

5. Baird AS. Pelvic actinomycosis: still a cause for concern. J Fam Plann Reprod Health Care 2005;31:73-4. 